\title{
The Beilby Layer
}

\author{
By G. I. Finch and A. G. Quarrell
}

$\mathrm{T}$ HE nature of polish has aroused considerable interest for nearly three centuries. Thus Hooke $^{1}$, in 1665, showed that grinding merely forms grooves, the fineness of which is determined by the grain-size of the abrasive, and from this he argued that the effect of a polishing powder was simply to cut very fine grooves. Herschel ${ }^{2}$ supported this hypothesis in that he regarded polishing as equivalent to a grinding action producing grooves of such fineness as to be invisible under the microscope.

This view was generally accepted until 1901, when Rayleigh ${ }^{3}$ pointed out the difference in effect produced by the same powder according to the nature of the backing material which served to force the polishing powder into contact with the surface. Thus he showed that only a matt finish could be obtained when a fine emery powder was backed by a glass sheet, whilst a pitch backing enabled a polish to be achieved. Rayleigh concluded that with a yielding support, such as pitch, the material is worn away almost molecularly. Between 1902 and 1921, the late Sir George Beilby ${ }^{4}$ made a close microscopic study of the effects produced by polishing a wide range of substances, and came to the conclusion that polish was the result of a flowing of the material, whereby the surface became coated with an amorphous layer. Among other striking experiments, he polished an antimony surface which had previously been ground with finest emery paper. Beyond occasional circular holes with smooth, rounded edges, the appearance of which was consistent with viscous flow of the antimony surface, no trace of the grinding furrows could be detected in the polished surface. Etching, however, removed the polished layer and restored to view the furrows left by the emery.

Beilby also postulated the formation of an amorphous layer whenever two crystal faces are rubbed together. Thus, he explained the hardening of metals produced by cold-working as being due to the formation between sets of slip planes of an amorphous cementing layer inhibiting further slip. It was this extension of Beilby's theory which first attracted most attention and led to its being vigorously attacked, particularly by Continental workers. Benedicks ${ }^{5}$, in particular, in refusing to accept the idea of an amorphous surface layer, reiterated his belief in the crystalline nature of the polish layer and suggested, indeed, that Beilby's results really supported this older view. To account for the hardening of metals, Benedicks postulated a process of multiple twinning leading to an interlocking of the crystals, whilst Tammann ${ }^{6}$ maintained that a repeated cleavage would suffice to account for the known phenomena without the assumption of the formation of an amorphous material. It was also suggested that the mechanical production of a vitreous layer under conditions in which only the crystalline state should be stable was improbable; but Desch? ${ }^{2}$, who was an early supporter of Beilby's views, has pointed out that this objection does not take account of the fact that the laws of heterogeneous equilibrium as usually expressed are only applicable when the pressure considered is of the hydrostatic kind, and quite different conditions may obtain when unsymmetrical pressure is applied. $\mathrm{He}$ also believed that Tammann's explanation, which en. visaged the formation of intercrystalline cavities, was unlikely, whilst Gough ${ }^{8}$ rendered Benedicks' hypothesis untenable by showing that single crystals of face-centred cubic metals did not necessarily twin even though they hardened on working.

Desch $^{7}$ has enumerated a number of properties of the polish surface which on the whole appear to support Beilby's hypothesis. Thus the polish layer displays greater chemical activity and is anodic to the crystalline material. The density is less than for the crystalline substance, which would be expected since a random arrangement occupies a greater space than a regular packing. On polishing a metal, the electrical conductivity is diminished and the hardness increased.

Thomson' was the first to take advantage of the new technique of electron diffraction in order to throw fresh light upon the structure of the polish layer. X-rays are unsuitable for this purpose because they are scattered in the main by extranuclear electrons, with the result that, owing to deep penetration, any information they might have given about the structure of the surface film is masked by the effects produced by the underlying material. Electrons, however, are scattered almost wholly by the nucleus, and the penetration is therefore slight. Hence any structural evidence obtained by the scattering of electrons from a surface is restricted to the immediate surface film. Thomson was unable to obtain patterns from polished surfaces of metals such as gold, iron, lead, 
aluminium and copper, and attributed this to the surface being covered with an amorphous layer resembling a super-cooled liquid.

A more detailed examination was carried out by French ${ }^{10}$, who followed by electron diffraction the change in structure with increasing degree of polish. He found that the sharp rings given by the originally crystalline surface became more and more blurred as the polishing progressed until, with the highest polish, the pattern consisted of two indistinct broad haloes on a diffuse background. French concluded that the ring broadening was due to a decrease in crystal size and that the breadth of the haloes in the final polish pattern could be explained by supposing either that the crystals had been reduced in size until they contained only a few unit cells, or that the process had gone so far as to destroy all semblance to a crystalline structure, leaving an amorphous film in which the limiting distance of atomic approach was the only orderly feature. Such a structure should give a pattern similar to that afforded by a gas or liquid, the halo radii depending upon the closest distance of atomic approach ; and using Wierl's ${ }^{11}$ relationship, French found by calculation halo radii agreeing fairly well with those of his polish patterns.

Randall and Rooksby ${ }^{12}$, in criticising these conclusions, suggested that the haloes obtained by French were in effect the two most prominent normal pattern rings blurred by reduction in crystal size, such blurring and consequent increase in background leading to the disappearance of all other rings. They attributed any discrepancy between ring and halo radii to the effect postulated on theoretical grounds by Lennard-Jones, according to which the lattice parameters of small crystals should differ from those of larger crystals. Randall and Rooksby based their criticisms mainly upon a series of X-ray patterns of graphite and various carbons; but it may be pointed out that whereas only two of the graphite pattern rings are intense, the crystalline surfaces of the metals studied by French give rise to several prominent rings, though the corresponding polish patterns only contain two haloes.

Germer ${ }^{13}$, who maintained that the polish layer was crystalline, obtained merely a general scattering from polished surfaces and attributed this to the surface being so levelled that the electrons entered and left through the same crystal face, and ho expressed the view that, with a comparatively slight inclination of the reflecting faces to the surface, an inner potential of 15 volts would, in the rase of nickel, for example, suffice to merge all the rings into a generally diffuse background. It is difficult to see how this result can be arrived at; furthermore, Germer's view does not take into consideration the existence of the haloes in French's patterns.

Kirchner ${ }^{14}$ regarded the polished surface as polycrystalline and attributed French's results to a specular reflection, the slight displacement of the maxima being due to refraction. In recalling Thomson's view that reflection from a polycrystalline surface is essentially a case of transmission through crystallites projecting above the surface, Kirchner pointed out that broadening of the rings with increasing degree of polish is not necessarily evidence of a reduction in crystal size, but may be equally well ascribed to a levelling of the surface such as to reduce the extent to which the crystals project above it. This effect should lead to a broadening of the rings until groups of neighbouring rings merged together to form ill-defined haloes.

Raether ${ }^{15}$, who had been associated with Kirchner in his earlier experiments, later gave an account of the structural changes observed during the mechanical working of surfaces. Polished, burnished and hammered iron, nickel, copper, silver, cadmium and gold surfaces all gave the characteris. tic halo pattern observed by French. According to Kirchner's explanation, the inner halo should lie between the 111 and 200 diffractions in the case of face-centred cubic metals; but although Raether found that this was so with gold and silver, for metals of lower atomic number such as nickel and copper the halo lay inside the 111 ring. The atomic separations calculated from the two halo radii by Prin's ${ }^{16}$ intensity distribution function for a liquid agree so satisfactorily as to support the view that the haloes are due to diffraction by a monatomic liquid. He therefore concluded that not only polishing, but also burnishing and ham. mering, cause a superficial layer, between $10 \mathrm{~A}$. and $100 \mathrm{~A}$. thick, either to flow or to break up into crystallites containing only a few unit cells, which are so pressed together as to give a compact structure in which the metal atoms are in densest packing with a mean distance of atomic approach.

Raether also examined polished single crystal faces of various non-conductors such as rocksalt, calcite, fluorite and pyrites, and obtained patterns which were typical either of reflection from the single crystal face or transmission through projecting crystal fragments integral with the main single crystal or through loose fragments on the crystal face. The halo pattern, however, never appeared. Hopkins ${ }^{17}$ also failed to obtain any evidence of a vitreous layer on polished calcite.

Darbyshire and Dixit ${ }^{18}$ extended French's experiments, particularly to structures other than cubic, and also obtained halo patterns with a ratio of the corresponding spacings of 0.55 as previously found by Raether. The interatomic distances calculated from the halo radii by 
Ehrenfest's equation compared well with those given by Keesom's relationship in the case of zinc, gold, silver, molybdenum, copper, chromium, silicon and selenium ; but were too low in the case of bismuth, antimony, tellurium, cadmium and lead, sometimes by as much as 20 per cent. But these metals, and also tin which Miwa ${ }^{19}$ found to behave similarly, are exceptional in that, as has been pointed out by Hume-Rothery ${ }^{20}$, ionisation is probably more or less incomplete in the solid. Darbyshire and Dixit concluded that all their halo patterns were due to the amorphous condition of the polish layers. On the other hand, Miwa, whilst accepting the vitreous layer idea in the case of copper, silver, chromium, iron, cobalt, nickel and zinc, attributed the patterns of abnormally large halo radii obtained from polished tin, cadmium and antimony to crystallites of such minuteness as to have very low resolving power. In support of this view, however, Miwa relies largely upon an antimony film obtained by condensation in vacuo being amorphous, though his corresponding pattern affords clear evidence of its being composed of exceedingly small crystals.

Kirchner ${ }^{21}$, in still adhering to the view that the polish layer is crystalline, bases his objections to Beilby's theory on experiments in which he has found that evaporated metal films often give halo patterns by reflection but sharp rings by transmission, and he ascribes this to a difference in the grain size effective in diffracting the beam. Thomson ${ }^{22}$, however, has pointed out a difficulty in accepting this explanation in so far as relates to the patterns from polished metals. Since there is no appreciable effect of refraction, the surfaces through which the electrons pass must make angles exceeding about $5^{\circ}$ with the electron beam. Taking the effective penetration of electrons as $4 \times 10^{-6} \mathrm{~cm}$. and the angle as $5^{\circ}$, the depth of the part of the crystal affected would be at least $2 \times 10^{-7} \mathrm{~cm}$., and this would be sufficient to give very much sharper rings than are, in fact, observed from a polished surface.

Thus although at this stage the balance seemed on the whole to incline towards Beilby's view of the nature of polish on metals, the issue was still in doubt when Finch, Quarrell and Roebuck ${ }^{23}$ discovered that the polish layer has the peculiar property, not possessed by the corresponding crystalline metal surface, of being able to dissolve crystals of a foreign metal at room temperature. Their experiments consisted in forming a crystalline metal film on a cool metal substrate by condensation in the evacuated electron diffraction camera. During deposition, the development of the pattern was observed on the fluorescent screen and the subsequent changes followed. The substrate was either highly polished or consisted of a previously polished surface which had been suitably etched to expose a polycrystalline surface. The polished surface gave no coherent pattern when the initial treatment had consisted of a vigorous high-speed buffing, though specimens polished entirely by hand gave typical halo patterns. The absence of haloes in the case of the buffed specimens was probably due, as Thomson ${ }^{24}$ has suggested, to a slight waviness of the surface producing so much further blurring due to variable refraction as to lead to their virtually complete submergence in the general background.

It was observed by Finch, Quarrell and Roebuck that immediately upon deposition of a zinc film on polished copper, a brilliant and well-defined diffraction pattern characteristic of crystalline zine was obtained which, however, rapidly faded, until after a few seconds no vestige of the pattern re. mained, thus showing that the crystalline structure had been destroyed. In one experiment, twelve successive zinc deposits were flashed over, and with each layer, except the last, the diffraction pattern vanished at a rate decreasing with each successive deposit. Thus the pattern yielded by the last layer but one was still faintly visible four minutes after deposition, but had completely vanished after five minutes, whilst after an initial period of weakening the pattern from the last deposit remained unchanged, even after four hours. Evidently the zinc layers had been dissolved by the polished copper surface and formed a solution which, with the final zinc layer, became saturated. On the other hand, a single zinc film deposited on an etched, that is crystalline, copper substrate gave a pattern which showed neither loss in brilliancy nor other change in appearance during the $1 \frac{1}{2}$ hours for which it was observed. Similar results were obtained with zinc, lead, silver, tin and later with cadmium and lithium deposited on polished and etched substrates of copper, iron, zinc, lead and gold. Finch, Quarrell and Wilman ${ }^{25}$ also found that whilst electrodeposition during 30 seconds at $0.1 \mathrm{amp} . / \mathrm{dem} .^{2}$ sufficed to form a permanent crystalline zinc layer on crystalline copper, three minutes were necessary if a polished copper electrode were used as substrate.

Just as the persistence of the diffraction pattern from the deposit on an etched substrate is evidence of the permanence of its crystal structure, so is the disappearance of the pattern when a polished substrate is used proof of the loss of erystalline structure in the deposit; and we are left with the conclusion that the polish layer, unlike the crystal. line surface, readily dissolves metal crystals and in so doing exhibits a property typical of the metal in the liquid state. Thus the evidence in support of Beilby's conception of the vitreous nature of the polish layer seems conclusive. 
Hopkins ${ }^{26}$ and Lees ${ }^{27}$ have recently measured the thickness of the Beilby layer on polished metals. Hopkins thinned the polish layer by cathodic sputtering under conditions permitting of measuring the rate of removal, and he found that a handpolished gold surface gave a pattern characteristic of crystalline gold after a layer of about $30 \mathrm{~A}$. had been removed by sputtering. Lees, in working with gold and copper surfaces, reduced the polish layer by electrolytic etching and arrived at similar estimates of thickness, and also concluded that the polish film was supported by a layer of crystals orientated by the polishing action.

It is natural to suppose that the depth of the Beilby layer is influenced by the vigour of the polishing action, and several observations have been made which confirm this. Thus it is not easy to demonstrate the solution of zinc erystals in a lightly hand-polished copper surface, though the effect is so striking when the final polishing is preceded by a vigorous machine buffing. Also, Finch, Quarrell and Wilman ${ }^{25}$ have examined the working surfaces of four aeroplane engine cylinder sleeves. Two were honed and ready for service whilst the others had been run-in for 40 hours and 140 hours respectively. After removal of the protective grease layer by washing with petrol-ether, the external and internal surfaces of the virgin sleeves both yielded patterns characteristic of a random crystal structure and in which $\alpha$-iron rings were prominent. The run-in surfaces, on the other hand, after degreasing gave the halo pattern typical of the Beilby layer. The thickness of this layer was such that although a single light stroke with No. 000 emery paper sufficed to break through the Beilby layer on a hand-polished steel specimen, several such abrasions were necessary before the haloes gave way to the normal ring patterns of the virgin sleeve surfaces. Thus it seems that the process of running-in an internal combustion engine can be likened to a vigorous polishing action, in that it consists in the formation on the working surfaces of an amorphous Beilby layer of considerable depth.

\footnotetext{
1 Hooke, "Micrographia", Observation II (1665).

2 Herschel, quoted by Rayleigh, Proc. Roy. Inst., 16, 563 (1901).

3 Rayleigh, Proc. Roy. Inst., 18, 563 (1901);

Beilby, "Aggregation and Flow of Solids", Macmillan (1921).

Benedicks, Rev. Met., 19, 505 (1922).

- Tammann, $Z$. anorg. Chem., 113, 163 (1920).

? Desch, "The Chemistry of Solids", p. 164, Cornell University Press (1934).

${ }^{B}$ Gough, Aeronautical Research Committee, Rep. and Mem., 1432 1930).

Thomson, Proc. Roy. Soc., A, 128, 649 (1930)

${ }_{10}$ French, Proc. Roy. Soc., A, 140, 637 (1933).

${ }_{11}$ Wierl, Ann. Physik, 8, 521 (1931)

12 Randall and Rooksby, NATURE, 129, 280 (1932).

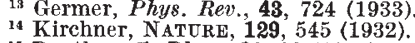

15 Kaether, $Z$. Phys., 86, 82 (1933).

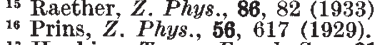

18 Hopkins, Trans. Farad, Soc., 31, 1127 (1935). ${ }_{18}$ Darbyshire and Dixit, Phil. Mag., 108, 961 (1933).

19 Miwa, Sci. Rep. Tôhoku Imp. Univ., 24, 222 (1935).

${ }_{20}$ Miwa, Sci. Rep. Tôhoku Imp. Univ., 24, 222 (1935). (1931)

${ }_{21}$ Kirchner, Trans. Farad. Soc., 31, 1114 (1935).

${ }^{22}$ Thomson, Trans. Farad. Soc., 31, 1116 (1935).

${ }_{23}$ Finch, Quarrell and Roebuck, Proc. Roy. Soc., A, 145, 676 (1934). 24 Thomson, Phil. Mag., 18, 640 (1934).

${ }^{25}$ Finch, Quarrell and Wilman, Trans. Farad. Soc., 31, 1077 (1935).

${ }^{26}$ Hopkins, Trans. Farad. Soc., 31, 1095 (1935)

27 Jees, Trans. Farad. Soc., 31, 1102 (1935).
}

\section{Metallic Wear in the Presence of Lubricants}

$\mathrm{T}$ HE sliding of one metal surface over another without the occurrence of an undue amount of wear is a problem which arises in every branch of engineering. Successful lubrication and the maintenance of a bearing surface depend upon a wide variety of factors, including those of a mechanical, a metallurgical and a chemical nature. Wear is largely determined by the affinity of one metal for another, the fouling or adhesion of one to the other leading to a roughness; the extent of this roughening and of the consequent wear is determined by the continuity of the film of lubricant separating the two metals, this continuity in turn depending upon the nature of the lubricant and the load it can support without rupture of the film. Our knowledge of lubricated surfaces was extended in 1932 by the work of Parish and Cammen in America, who found that below the film of lubricant there exists a small quantity of oil in the cavities or pores of the surface layers of the metal. Since this work was carried out, however, very little attention seems to have been given to the problems of metallic wear, despite their great importance to engineers, metallurgists, chemists and physicists.

Major interest in metallic wear centres to-day upon the problems involved in the successful running of automobile and aeroplane engines. Here bearing pressures and temperatures are high, and every endeavour must be made to provide bearing surfaces which will give a satisfactory service life under the stringent conditions imposed. Despite the existence of one or two contradictory examples, the old-established concept of a duplex structure, of hard particles embedded in a softer matrix, still prevails, and shows no indication of being superseded. There is, however, one other aspect of metallic wear upon which a certain amount of attention has recently been focused; this is associated with problems of die-fouling in 